\title{
Simulation, identification and dynamic control of the luminaire of the synthesized spectrum
}

\author{
Alexander Lukyanov*, Danila Donskoy, and Miroslav Vernezi \\ Don State Technical University, Rostov-on-Don, Russia
}

\begin{abstract}
We consider the mathematical problem of the best (in the sense of OLS) approximation of the spectrum of sunlight modified by passing through thin porous environment, light from several sources with functionally or tabular given spectral density. The problem differs from the one known for the formation of light of the "white" spectrum by the optimization of the integral index of filling the spectral range and taking into account the real spectral function of the sources. Method for forming of the specified spectrum led managed agro-lighting.
\end{abstract}

\section{Introduction}

A number of tasks agro-photonic requires the formation of irradiation of a given spectrum for the stimulation of the growth processes of plants at different stages of development from seed germination. In a number of works [5-9] the influence of the spectral composition of light on the germination of seeds of vegetable and green culture was noted. Aware of the impact of the spectral components of the light on the phenotype of seedlings and plants [7]: the predominance of red light leads to thickening of the stalk of a plant, the predominance of blue - elongation, etc. a Number of authors describes the influence of the light spectrum and photoperiod on the change of vegetation stages: the transition to flowering, fruiting, etc. Not fully explored influence diurnal variation of the spectrum of light for growing process. Changes in the spectral composition of sunlight during its penetration into the soil were studied [1-3]. Also note the spectral selectivity of plant vegetation processes [4], Fig. 1. Providing lighting of farming culture with light of optimized spectral composition, along with the above features, can also lead to energy savings, and increase productivity at the same energy costs.

\footnotetext{
*Corresponding author: alexlukjanov1998@gmail.com; dand22@bk.ru
} 


\section{Approximations of a continuous spectrum by a spectrum of several sources}

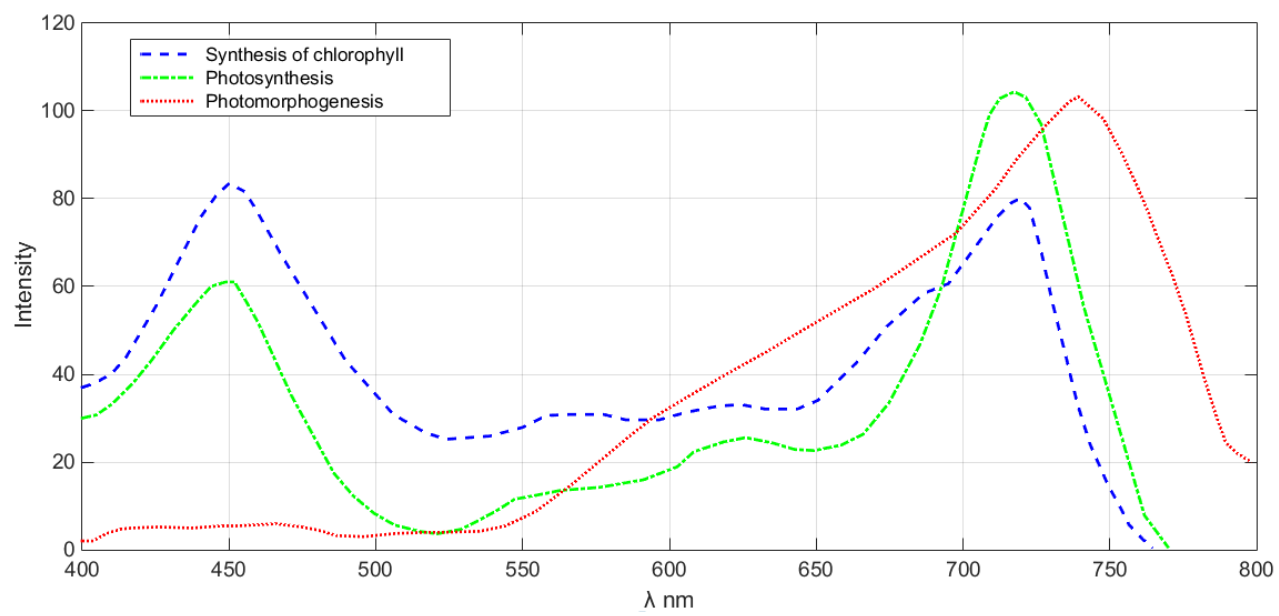

Fig. 1. Spectral sensitivity of vegetation processes. On materials [4]

Consider the spectrum of solar radiation (Fig. 2)

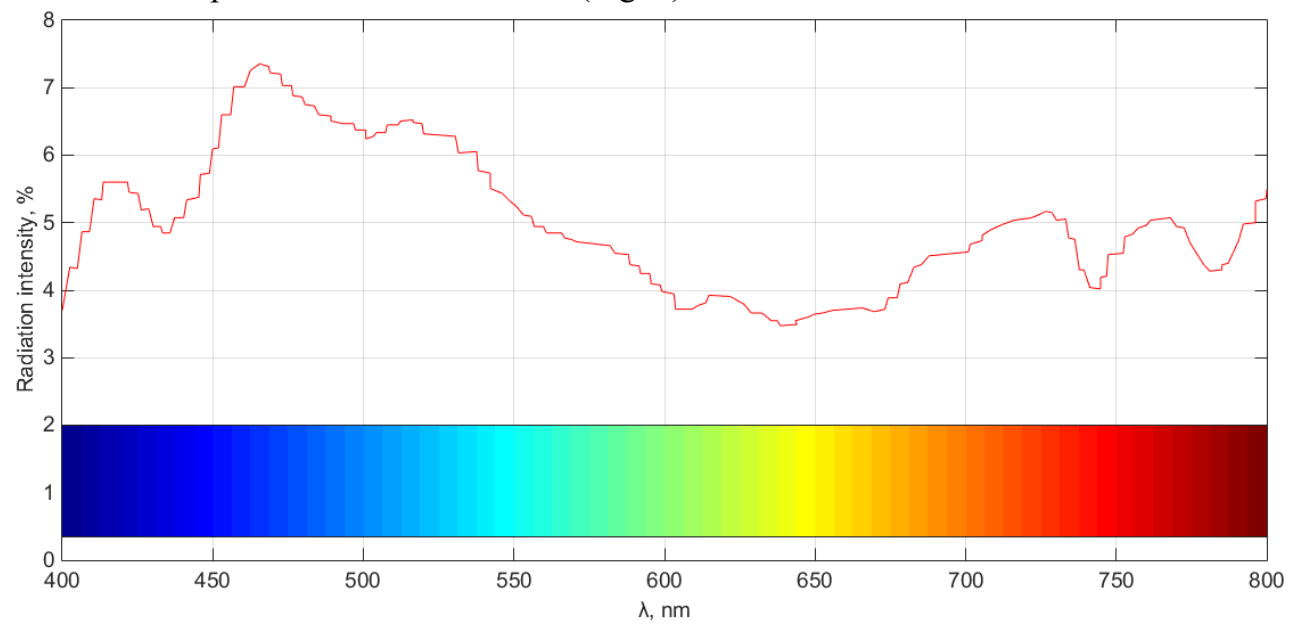

Fig. 2. An example of the solar spectrum on a low-cloud day in winter.

In [1] the data on changes in the spectral composition of sunlight after passing through the soil layers of different thickness and composition are presented (Fig. 3). There is a radical reduction of high-frequency spectrum components (blue-green) while maintaining lowfrequency (red and near IR). 


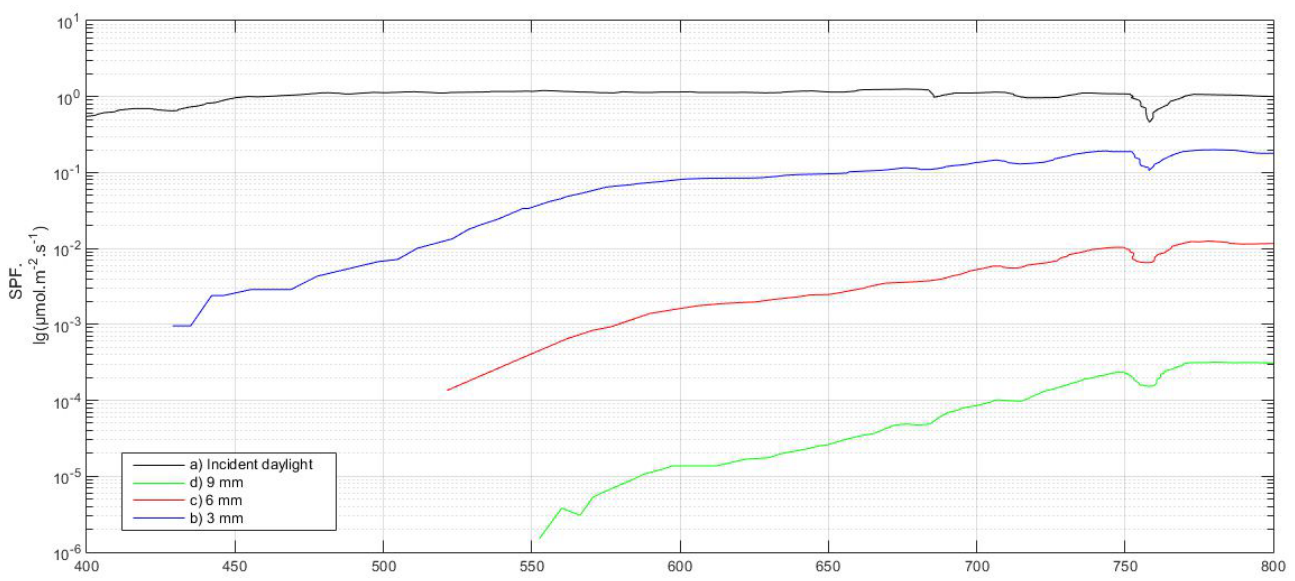

Fig. 3. An example of spectra of changed sunlight on a clear summer day after passing through soil layers. (based on the materials [1]). (a) incident daylight; (b), (c) and (d) are daylight transmitted through 3, 6 and $9 \mathrm{~mm}$ of coarse sand, respectively.

\section{Formulation and analytical solution of the optimal spectrum approximation problem}

Consider the given spectral density function $S(\lambda)$, which must be approximated by a linear combination of several available functions of the spectral density of light sources $S_{1}(\lambda)$ $S_{n}(\lambda):$

$$
\hat{S}(\lambda)=k_{1} S_{1}(\lambda)+k_{2} S_{2}(\lambda)+\ldots+k_{n} S_{n}(\lambda)
$$

At the same time, we will consider the optimization functionality in the form of:

$$
J\left(k_{1}, k_{2}, \ldots k_{n}\right)=\int_{\lambda_{1}}^{\lambda_{2}}\left(S(\lambda)-\sum_{i=1}^{n} k_{i} S_{i}(\lambda)\right)^{2} d \lambda \underset{k_{1} \ldots k_{n}}{\longrightarrow} \min
$$

Integration means that the optimization will be carried out not by the envelope $S(\lambda)$, but by the total irradiation power in the spectral range from $\lambda_{1}$ to $\lambda_{2}$.

The optimal values of the coefficients $k_{1}, k_{2}, \ldots k_{n}$ are achieved by solving a system of equations:

$$
\frac{J}{\partial k_{i}}=0, \quad i=1, n
$$

The calculated derivatives have the form:

$$
\frac{J}{\partial k_{i}}=2 \int_{\lambda_{1}}^{\lambda_{2}}\left(S(\lambda)-\sum_{j=1}^{n} k_{j} S_{j}(\lambda)\right)\left(-S_{i}(\lambda)\right) d \lambda
$$

A fter opening the brackets and dividing the sum in the integral into summands, we obtain: 


$$
\frac{J}{\partial k_{i}}=\int_{\lambda_{1}}^{\lambda_{2}} S(\lambda) S_{i}(\lambda) d \lambda+\sum_{j=1}^{n} k_{j} \int_{\lambda_{1}}^{\lambda_{2}} S_{i}(\lambda) S_{j}(\lambda) d \lambda
$$

The system of linear equations $\mathbf{A}_{S} \mathbf{K}=\mathbf{B}_{S}$ for finding the optimal values collected in the vector of estimates $\mathbf{K}=\left(k_{1}, k_{2}, \ldots k_{n}\right)$ has the following components:

$$
\mathbf{A}_{S}=\left(\begin{array}{ccc}
\int_{\lambda_{1}}^{\lambda_{2}} S_{1}(\lambda) S_{1}(\lambda) d \lambda & \cdots & \int_{\lambda_{1}}^{\lambda_{2}} S_{1}(\lambda) S_{n}(\lambda) d \lambda \\
\vdots & \ddots & \vdots \\
\int_{\lambda_{1}}^{\lambda_{2}} S_{n}(\lambda) S_{1}(\lambda) d \lambda & \cdots & \int_{\lambda_{1}}^{\lambda_{2}} S_{n}(\lambda) S_{n}(\lambda) d \lambda
\end{array}\right) \quad \mathbf{B}_{S}=\left(\begin{array}{c}
\int_{\lambda_{1}}^{\lambda_{2}} S(\lambda) S_{1}(\lambda) d \lambda \\
\vdots \\
\int_{\lambda_{1}}^{\lambda_{2}} S(\lambda) S_{n}(\lambda) d \lambda
\end{array}\right)
$$

Integral components of expressions can be easily calculated by given functions $S(\lambda)$ and $S_{1}(\lambda)-S_{n}(\lambda)$ this makes it easy to calculate the components of the vector $\mathbf{K}$ :

$$
\mathbf{K}=\left(A_{s}^{T} A_{s}\right)^{(-1)} A_{s}^{T} B_{s}
$$

\section{Examples of approximation of the spectrum with the spectra of the LEDs}

According to the article [5] the required relation of colors in the spectrum of the lamp is given as $\mathrm{R}: \mathrm{G}: \mathrm{B}=33: 33: 34$. However, this ratio is speculative, and does not take into account the real, non-point spectrum of modern diodes, and does not take into account the possibility of variation of spectral components at different stages of vegetative development of plants. For fig. 4 shows an example of the approximation of the spectrum of sunlight using the four types of LEDs: R, G, B and ColdWhite.

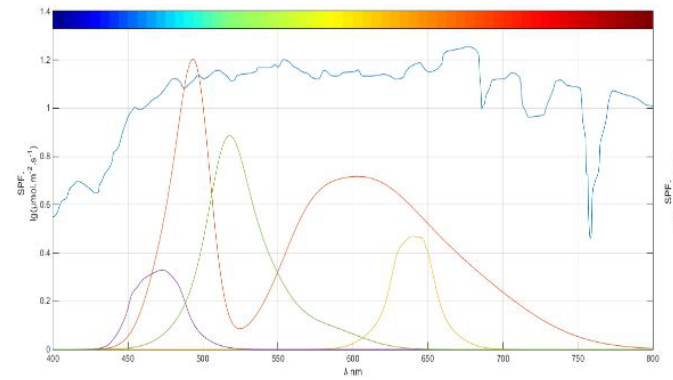

a

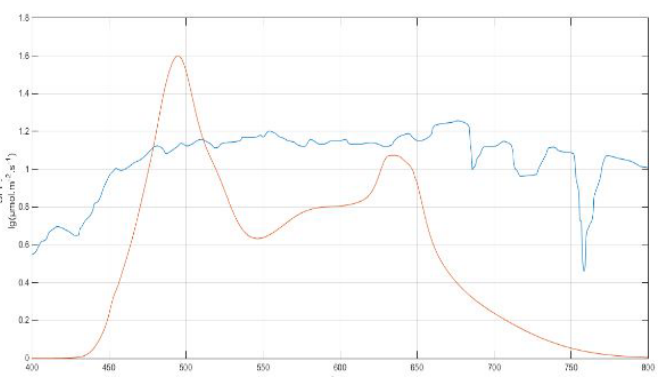

b

Fig. 4. Decomposition of RGBW led colors by spectrum and comparison with sunlight (a) and total approximation (b).

\section{Conclusion}

The paper presents an algorithm that allows to identify the coefficients of approximation of the required spectrum of discrete light sources, for example, the use of LEDs. This takes into account the real spectral density of light sources, which can be set functionally or tabular. The estimate is optimal in the sense of minimizing the error square. The algorithm can be used to specify fixed intensities of light of LEDs of different spectral composition in agro- 
lighting synthetic spectrum and to determine the initial values of the intensity of the luminosity of the LEDs with multichannel automatic control of the spectral composition of agro-lighting.

\section{References}

1. D. Bliss, H. Smith, Plant Cell and Environment, 8(7), 475 (2006) doi: 10.1111/j.13653040.1985.tb01683.x

2. M. Tester, C. Morris, Plant Cell and Environment, 10(4), 281 (2006) doi: I10.1111/j.1365-3040.1987.tb01607.x

3. D. F. Mandoli, G. A. Ford, L. J. Waldron, J. A. Nemson, W. R. Briggs, Plant, Cell \& Environment, 13, 3, 287 (1990), doi: 10.1111/j.1365-3040.1990.tb01313.x

4. N. S. Kononov, K. Timiryazev, LLC "NPK LEDART" On the materials of the report at the exhibition "Golden Autumn 2013"

5. A.V. Patsukov, A. P. Mishanov, S. A. Rakutko, A. E. Markov, V. N. Sudachenko, Svitlotekhnika, UDC: 628.941.8: 581.14

6. S. Pongnumkul, P. Chaovalit, and N. Surasvadi, Journal of Sensors, 2015, 195308, 18 http://dx.doi.org/10.1155/2015/195308

7. Optimizing LED Lighting for plants. https://bioslighting.com/agriculture-lighting/

8. Artificial lighting in agriculture http://www.valoya.com/wpcontent/uploads/2018/03/English-Version_Artificial-lighting-in-agriculture.pdf

9. VALOYA LED GROW LIGHTS http://www.valoya.com/category/blog/ 